Revisão

\title{
Trombocitopenia induzida por heparina
}

Fernanda Longhi ${ }^{1}$

Dani Laks ${ }^{2}$

Nelson G.N. Kalil ${ }^{3}$
O objetivo deste relato é fazer uma revisão a fim de obter informações atualizadas sobre trombocitopenia induzida por heparina, suas manifestaçôes clínicas, seu diagnóstico e seu manejo terapêutico.

Após, concluímos que a trombocitopenia induzida por heparina é uma complicação comum em pacientes submetidos a tratamento com heparina, indiferentemente da doença de base. Complicações trombóticas potencialmente fatais têm sido descritas. Por essa razão, se houver suspeita de trombocitopenia induzida por heparina, uma abordagem adequada incluindo suspensão precoce da heparina é mandatória. Produtos que substituem a heparina incluem birudina e danaparóide sódico. Heparina de baixo peso molecular é contraindicada.

Rev.bras.hematol.hemoter., 2001, 23(2): 93-99

Palavras-chave: Trombocitopenia, heparina, manifestaçôes clínicas, tratamento, birudina, danaparóide sódico

\section{Introdução}

A heparina é uma droga com ação antitrombótica amplamente utilizada desde a década de 1930 (1, 2). Há vários efeitos adversos relacionados ao uso da heparina: hemorragias, osteoporose, eosinofilia, reações cutâneas, alopécia, alteração dos testes de função hepática e hipercalemia ocasional $(2,3)$. Entretanto, um dos mais importantes efeitos adversos da heparina é a trombocitopenia (1, 2, 3, 4). A trombocitopenia induzida por heparina (TIH) apresenta duas formas: a TIH tipo I, menos severa, que ocorre em cerca de 20 a $25 \%$ dos pacientes tratados com heparina, e a TIH tipo
II, mais severa, que ocorre em 2 a $5 \%$ dos pacientes que recebem heparina $(1,2,5,6)$. A TIH tipo II está relacionada a eventos trombóticos com potencial risco de vida $(7,8)$.

\section{Trombocitopenia induzida por heparina}

O primeiro relato de trombocitopenia relacionada ao uso de heparina foi feito em 1942 por Copley e Robb após a realização de estudos com cães. O desenvolvimento de complicações trombóticas em pacientes recebendo heparina foi descrito em 1958. Entretanto, a relação entre o desenvolvimento de trombocitopenia e a ocorrência de tromboembolismo em pacientes

1 - Médica Residente do Hospital São Lucas da Pontifícia Universidade Católica - RS

2 - Médico Hematologista do Hospital São Lucas da Pontifícia Universidade Católica - RS

3 - Médico Oncologista do Hospital São Lucas da Pontifícia Universidade Católica - RS

Correspondência: Fernanda Longhi

Rua Tamoio, 2260. 92120-002. Canoas. RS

Fone: (51) 472-3749. E-mail: ferlonghi@conex.com.br 
tratados com heparina só foi notada no início dos anos 70 (1).

A trombocitopenia induzida por heparina (TIH) é uma complicação comum em pacientes que recebem terapia com heparina nãofracionada $(3,5,9)$. Aqueles que recebem heparina de baixo peso molecular apresentam uma ocorrência menor de TIH $(5,6)$. Em sua forma mais severa, a TIH está associada a um alto risco de eventos trombóticos com potencial risco de vida $(4,5,7,8,9)$.

Pacientes que apresentam uma queda de 30 a 50\% na contagem de plaquetas durante o tratamento com heparina, mesmo que não apresentem trombocitopenia, também possuem risco aumentado de desenvolver eventos trombóticos $(2,6,10)$. Por isso, pacientes recebendo heparina que apresentem complicações trombóticas, mesmo sem trombocitopenia, devem ser investigados para TIH.

\section{Classificação}

Há dois tipos distintos de TIH: tipo I e tipo II. A TIH tipo I é a forma menos severa e também a mais freqüente, ocorrendo em aproximadamente 20 a $25 \%$ dos pacientes tratados com heparina (5). É caracterizada por uma trombocitopenia leve que geralmente inicia precocemente após o uso da heparina, mas que pode ocorrer mais tardiamente. Raramente a contagem de plaquetas atinge valores menores que $100 \times 10^{\%} / \mathrm{dl}^{3}$ (11). Não há eventos clínicos associados à TIH tipo I, que provavelmente é causada pelo efeito direto da heparina nas plaquetas, resultando na sua agregação $(1,4,5,11)$.

A TIH tipo II ocorre em 2 a 5\% dos pacientes que recebem heparina $(1,2,4,5,6)$, incluindo aqueles que recebem baixas doses da droga e, até mesmo, aqueles que recebem heparina apenas para manter permeável a via de catéteres venosos (11). É caracterizada por uma trombocitopenia mais severa, freqüentemente menor que $100 \times 10^{9}$ plaquetas/dl, que ocorre de quatro a quatorze dias após o início da administração de heparina $(5,6,9,11,12)$. Entretanto, pode ocorrer antes desse período em pacientes que tenham sido expostos previamente à heparina nos últimos três meses $(4,6)$. Dos pacientes que desenvolvem TIH tipo II, cerca de 10\% desenvolverão eventos trombóticos $(7,8)$.

\section{Patogenia}

A patogenia da TIH tipo II é explicada por um mecanismo imune, em que os pacientes desenvolvem anticorpos heparina-dependentes no plasma. Na maioria dos pacientes esses anticorpos são da classe $\operatorname{IgG}(2,5,7,8,9,11$, 13). Anticorpos da classe IgA e/ou IgM também podem estar presentes em cerca de 25 a 35\% dos pacientes com TIH tipo II. Entretanto, raramente são encontrados isoladamente (11).

Uma vez presentes, os anticorpos promovem ativação plaquetária através dos seus receptores Fc. As plaquetas então liberam o chamado fator-4-plaquetário, uma proteína encontrada nos seus grânulos alfa que possui alta afinidade de ligação com a heparina $(5,7$, 9, 11). Essa ligação forma um complexo multimolecular heparina/fator-4-plaquetário (1, 6), que é o alvo antigênico dos anticorpos heparina-dependentes (5). A ligação dos anticorpos aos antígenos ocorre na superfície das plaquetas e na superfície das células endoteliais $(7,11)$, resultando em agregação e destruição plaquetária e em lesão das células endoteliais. Conseqüentemente, ocorre ativação da cascata da coagulação e aumento na síntese de trombina $(2,11,13)$. Todos esses fatores fazem aumentar o risco de complicações tromboembólicas $(11,13)$.

\section{Manifestações Clínicas}

As mais freqüentes complicações da TIH tipo II são tromboembolismos venosos, especialmente trombose venosa e embolia pulmonar $(5,6,9,11)$. As complicações arteriais geralmente envolvem as grandes artérias dos membros inferiores, levando à isquemia aguda das extremidades $(4,6)$.

Um estudo realizado por Warkentin e colaboradores (9) sugeriu que o tipo de complicação trombótica (arterial ou venosa) é influenciado pela situação clínica do paciente. Pacientes em pós-operatório, especialmente de cirurgia ortopédica, têm risco aumentado de trombose venosa, assim como pacientes com 
doença cardiovascular apresentam maior propensão a desenvolver trombose arterial (6).

Outras complicações arteriais relativamente comuns são acidente vascular encefálico e infarto agudo do miocárdio (4, 6). Infarto hemorrágico bilateral de adrenais e trombose de artéria renal são síndromes incomuns $(4,11)$.

\section{Métodos Diagnósticos}

Sempre que possível, o diagnóstico clínico de TIH tipo II deve ser confirmado através de testes laboratoriais capazes de detectar anticorpos heparina-dependentes ou antígenos heparina/fator-4-plaquetário. A confirmação de um diagnóstico clínico é importante para definir futuros tratamentos com heparina, mas não para decisão imediata, uma vez que há indicação de suspender a droga frente à suspeita de TIH (11).

Os métodos utilizados para detectar antígenos são métodos de imunoensaio, enquanto aqueles utilizados para detectar anticorpos são métodos funcionais (11).

O método de imunoensaio utilizado é o ELISA. Ele detecta a ligação dos anticorpos aos complexos multimoleculares heparina/fator-4plaquetário $(6,11)$. A sensibilidade do método ELISA é de aproximadamente 80 a $90 \%$ e, por isso, ele é capaz de detectar até mesmo ligação a anticorpos mais fracos, como IgA e IgM (11). Entretanto, o método pode ter uma baixa especificidade, apresentando resultados falsopositivo (6).

Os métodos funcionais medem a ativação plaquetária causada pelo anticorpo heparinadependente in vitro (11). O método padrãoouro utilizado para confirmar o diagnóstico de TIH tipo II é o que utiliza serotonina marcada com carbono 14 (2, 6, 9, 11). Os anticorpos heparina-dependentes causam ativação plaquetária e conseqüente liberação de serotonina marcada com carbono 14 dos grânulos plaquetários no momento em que o soro do paciente é incubado com plaquetas radiomarcadas de um doador normal em concentrações terapêuticas de heparina (11). Esse método é altamente sensível e específico para TIH tipo II, mas é utilizado freqüentemente apenas para pesquisas, uma vez que sua técnica despende muito tempo e requer o uso de uma substância radioativa $(6,11)$.

Outros métodos funcionais são o de ativação plaquetária induzida por heparina e o de agregação plaquetária.

No método de ativação plaquetária induzida por heparina o soro do paciente é colocado em quatro frascos contendo plaquetas lavadas de quatro doadores normais na presença de duas concentrações diferentes de heparina. É considerado um resultado positivo quando houver agregação plaquetária em pelo menos dois frascos. Essa técnica também possui alta sensibilidade e, além disso, utiliza menos tempo (11).

O teste de agregação plaquetária consiste em incubar o soro ou o plasma do paciente com plasma rico em plaquetas de doadores normais e heparina. A ocorrência de agregação plaquetária confirma o diagnóstico de TIH tipo II. Esse método é amplamente utilizado por ser rápido e tecnicamente simples. Embora possua baixa sensibilidade (entre 70 e 80\%), sua especificidade é alta (entre 80 e 100\%) $(8,12,14)$.

Métodos funcionais que utilizam citometria de fluxo também têm sido usados para detectar anticorpos heparina-dependentes $(1,11)$.

Como os testes laboratoriais nem sempre encontram-se disponíveis, o diagnóstico inicial de TIH tipo II e as decisões de tratamento devem ser baseados somente na apresentação clínica do efeito adverso $(4,6)$.

Uma vez que o número de pacientes que desenvolve anticorpos heparina-dependentes é maior do que o número de pacientes que desenvolve sintomas clínicos $(9,11)$, a realização de testes de screening para pacientes assintomáticos com plaquetopenia não é indicada atualmente (11).

\section{Manejo Terapêutico}

Frente à suspeita de TIH, é mandatória a suspensão imediata da heparina $(2,6,7,12$, 13). A contagem de plaquetas geralmente normaliza dentro de sete a dez dias após (4, 12). Entretanto, somente a sua suspensão não é a terapêutica apropriada $(12,15)$. Antes de optar pelo tratamento adequado, é importante saber o que deve ser evitado nos casos de TIH.

A heparina de baixo peso molecular não é 
recomendada para tratamento de TIH por apresentar reatividade cruzada in vitro com os anticorpos formados em mais de $90 \%$ dos casos $(6,9,13)$. Essa reatividade cruzada in vivo faz com que haja persistência da trombocitopenia nesses pacientes, assim como a ocorrência de novos ou recorrentes eventos trombóticos (14).

Anticoagulantes orais também não devem ser usados como substitutos da heparina nos pacientes com TIH. Eles possuem um lento início de ação e promovem uma queda dos níveis de proteína $\mathrm{C}$ que, associada ao aumento na geração de trombina já existente nesses pacientes, coloca-os em um risco maior de complicações tromboembólicas $(2,14)$. Seu uso tem sido associado à gangrena venosa de extremidades. A terapia com anticoagulante oral deve ser postergada até que a trombocitopenia tenha sido resolvida (6).

O ancrode é uma proteína extraída do veneno da cobra Agkistrodon rhodostroma que tem sido usado como um agente antitrombótico. Seu uso não é recomendado para tratamento de TIH tipo II porque ele não inibe a síntese de trombina e porque seu efeito antitrombótico é muito lento. Além disso, por ser uma proteína estranha, seu uso está relacionado a severas reações alérgicas $(2,14)$.

Devido à participação da trombina na patogênese da TIH, o tratamento primário dessa patologia deve incluir uma droga que reduza a síntese de trombina. Entre essas drogas encontram-se os inibidores diretos da trombina, a hirudina e o argatroban, e um inibidor indireto da trombina, o danaparóide $(6,14,15)$.

A hirudina é o mais potente e específico inibidor da trombina conhecido atualmente, sendo extraída das glândulas salivares de sanguessugas medicinais $(13,15)$. Ela liga-se à trombina com alta afinidade e especificidade, formando um complexo não covalente irreversível e, com isso, inibindo todas as funções proteolíticas da trombina (15).

Ao contrário da heparina, a hirudina não é inativada pelo fator-4-plaquetário $(12,13)$. Além disso, por apresentar estrutura química diferente da estrutura da heparina, não ocorre reatividade cruzada entre essas drogas (6).

A hirudina é metabolizada e excretada via renal. Por isso, os pacientes com insuficiência renal apresentam maior risco de acúmulo da droga e, conseqüentemente, de sangramento (6). A dose de hirudina deve ser ajustada nesses pacientes.

Várias formas comerciais de hirudina são manufaturadas através do emprego de biotecnologia recombinante (6). A lepirudina (Tabela 1) é uma hirudina recombinante dissulfatada (12), cujo tratamento está relacionado a uma rápida e sustentada recuperação na contagem de plaquetas e a um prolongamento suficiente do tempo de tromboplastina parcial ativada (KTTP) (13). Seu efeito adverso mais comum é sangramento. Outros efeitos incluem reações cutâneas, sepse, pneumonia e aumento de enzimas hepáticas (12). Além disso, o uso de lepirudina tem sido relacionado à formação de anticorpos IgG anti-

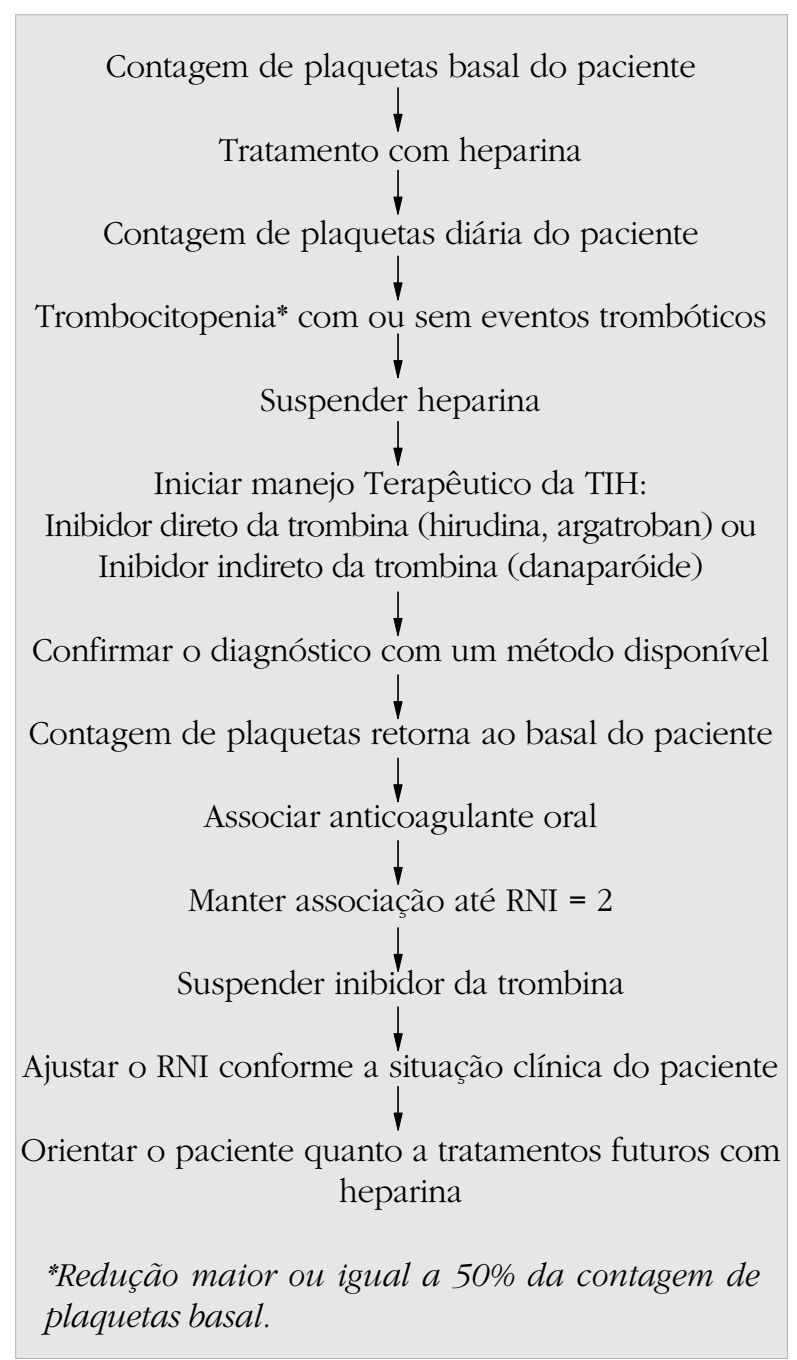

Figura 1. Manejo clínico da TIH 
hirudina em alguns pacientes que recebem a droga por mais de cinco dias. Nesses pacientes, há um aumento maior do KTTP (6).

A hirudina tem sido aprovada na Europa para o tratamento de TIH desde março de 1997 e, nos Estados Unidos, desde março de 1998. Atualmente, ela é a única droga aprovada nos Estados Unidos para o tratamento da TIH (6).

O argatroban é um inibidor sintético da trombina (12). Ele atua de forma reversível, direta e seletiva. Suas propriedades farmacológicas fazem com que ele atinja uma rápida eficácia terapêutica antitrombótica, com mínimo risco de sangramento e rápida restauração da hemostasia ao normal no momento da sua descontinuação (6).
A ligação reversível à trombina e a curta meia-vida do argatroban conferem a ele uma vantagem segura sobre a hirudina recombinante. Além disso, o argatroban é excretado via hepática, podendo ser usado em pacientes com insuficiência renal severa $(6,15,16)$.

O danaparóide sódico (Tabela 2) é um componente heparinóide semelhante à heparina de baixo peso molecular que tem sido usado mundialmente por mais de dez anos (6, 13). Seu componente ativo é isolado da mucosa intestinal do porco. Por isso, pacientes com hipersensibilidade a produtos suínos podem apresentar reações alérgicas com a medicação. Apesar de ser um agente relativamente seguro, apresenta reatividade cruzada in vitro com

Tabela 1. Dose de birudina recombinante (16)

Hirudina recombinante $\left(\right.$ Refludan $^{\circledR}$ )

Dose inicial

$0,4 \mathrm{mg} / \mathrm{kg}$ IV em bolus

Dose de manutenção

$0,15 \mathrm{mg} / \mathrm{kg} / \mathrm{h}$ (ajustar a dose para manter o KTTP entre $1,5-3$ vezes o valor normal)

KTTP - Tempo parcial de tromboplastina ativada

Tabela 2. Dose de danaparóide sódico (16)

Danaparóide sódico $\left(\right.$ Orgaran $\left.^{\circledR}\right) 1$ amp - 750 anti-Xa - 0,6 mL

Dose inicial

< $60 \mathrm{~kg}: 1.500$ UI IV em bolus

60-75 kg: 2.250 UI IV em bolus

75-90 kg: 3.000 UI IV em bolus

$>90$ kg: 3.750 UI IV em bolus

Dose de manutenção

$400 \mathrm{U} / \mathrm{h}$ durante 4 horas, seguido por $300 \mathrm{U} / \mathrm{h}$ durante 4 horas, seguido por 150-200 U/h

Tabela 3. Valores de RNI recomendados para pacientes em uso de anticoagulantes orais (17)

\begin{tabular}{|c|c|}
\hline Situação clínica do paciente & RNI \\
\hline Profilaxia de trombose venosa profunda & $2-3$ \\
Profilaxia de embolia pulmonar & $2-3$ \\
\hline Profilaxia de tromboembolismo sistêmico & \\
$-\quad$ fibrilação atrial & $2-3$ \\
$-\quad$ válvula cardíaca mecânica & $2,5-3,5$ \\
$-\quad$ válvula cardíaca biológica & $2-3$ \\
\hline
\end{tabular}


anticorpos antiplaquetários associados à heparina em 10 a 20\% dos casos $(14,15)$. Além disso, não possui antídoto disponível (2, 6, 13, 15). Hematomas epidurais podem ocorrem com o uso associado de heparinóides e anestesia epidural ou punção lombar, podendo resultar em paralisia de longa duração ou até permanente. O risco desse evento adverso é maior com o uso de catéter epidural para analgesia ou com o uso concomitante de drogas adicionais que afetam a hemostasia, tais como antiinflamatórios não-esteróides e antiagregantes plaquetários.

Os agentes antiagregantes plaquetários são capazes de promover um aumento na contagem de plaquetas em alguns pacientes com TIH, mas sua baixa eficácia contra o complexo antígeno/ anticorpo limitam seu uso como terapia adjuvante $(2,6,15)$.

Altas doses de imunoglobulina intravenosa são capazes de deslocar os anticorpos antiheparina das plaquetas, mas como essa droga não é um agente anticoagulante, ela somente é usada como terapia adjuvante $(6,14)$.

Após a normalização da contagem de plaquetas, pode ser iniciada a administração de anticoagulantes orais. A associação entre o anticoagulante oral e a droga escolhida para o tratamento da TIH deve ser mantida até que o RNI atinja 2. Após, pode ser reduzida gradualmente a dose da droga até que o RNI esteja adequado para a situação clínica do paciente (Tabela 3) para, então, suspendê-la $(13,17)$.

A plasmaferese tem sido usada em pacientes com TIH para ajudar a remover os complexos imunes presentes $(2,5,6)$. É um método terapêutico seguro que não interfere com outras modalidades de tratamento (5). Robinson e Lewis (5) realizaram um estudo com o objetivo de avaliar o papel da plasmaferese no tratamento da TIH. Concluíram que a plasmaferese pode ser útil, reduzindo a mortalidade, quando for realizada dentro de quatro dias após o início da trombocitopenia. Também sugerem que a ausência de resposta nos pacientes submetidos à plasmaferese após esse período deva ser conseqüência, em parte, ao maior tempo de exposição à heparina desses pacientes.
É importante lembrar que qualquer trombo complicado em pacientes com TIH deve ser tratado com protocolos standards de tratamento, evitando apenas o uso de heparina (6).

\section{Conclusão}

A TIH é uma complicação comum em pacientes submetidos a tratamento com heparina indiferentemente da doença de base. Complicações trombóticas potencialmente fatais têm sido descritas. Por essa razão, se houver suspeita de TIH, uma abordagem adequada incluindo a suspensão precoce da heparina é mandatória. Produtos que substituem a heparina incluem a hirudina e o danaparóide sódico. A heparina de baixo peso molecular é contra-indicada.

\section{Heparin-induced thrombocytopenia}

Fernanda Longhi, Dani Laks, Nelson G.N. Kalil

\section{Abstract}

The aim of this paper is to review current information about the clinical manifestations, diagnosis and management of heparin-induced thrombocytopenia. This was achieved by a bibliographic review using Public Medline and consulting Hematology textbooks.

From this study we concluded that heparininduced thrombocytopenia is a common complication of patients exposed to heparin therapy regardless of underlying conditions. Potentially fatal thrombotic complications have been reported. Therefore, if heparin-induced thrombocytopenia is suspected, an adequate approach including early beparin discontinuation is mandatory. Alternative products for beparin include birudin and danaparoid sodium. Low-molecular-weight heparin is contraindicated.

Rev.bras.hematol.hemoter., 2001, 23 (2): 93-99

Key words: Thrombocytopenia, heparin, clinical manifestations, therapy, birudin, danaparoid sodium 


\section{Referências Bibliográficas}

1. Ortel T.L. Heparin-induced thrombocytopenia. Semin Hematol 1998; 35 (4 suppl 5): 1-2.

2. Walenga J.M., Bick R.L. Heparin-induced thrombocytopenia, paradoxical thromboembolism, and other side effects of heparin therapy. Med Clin North Am 1998; 82 (3): 635-58.

3. Crowther M.A., Ginsberg J.S., Tollefsen D.M., Blinder M.A. Heparin. In: Hoffman R, Benz Jr EJ, Shattil SJ, et al. Hematology basic principles and practice. 3th ed. Philadelphia: Churchill Livingstone, 2000. p. 2046-56.

4. Warkentin T.E. Clinical presentation of beparin-induced thrombocytopenia. Semin Hematol 1998; 35 (4 suppl 5): 9-16.

5. Robinson J.A., Lewis B.E. Plasmapheresis in the management of heparin-induced thrombocytopenia. Semin Hematol 1999; 36 (1 suppl 1): 29-32.

6. Kelton J.G. The clinical management of heparin-induced thrombocytopenia. Semin Hematol 1999; 36 (1 suppl 1): 17-21.

7. Nand S., Wong W., Yuen B., et al. Heparininduced thrombocytopenia with thrombosis: incidence, analysis of risk factors, and clinical outcomes in 108 consecutive patients treated at a single institution. Am J Hematol 1997; 56: 12-6.

8. Suh J.S., Malik M.I., Aster R.H., Visentin G.P. Characterization of the humoral immune response in beparin-induced thrombocytopenia. Am J Hematol 1997; 54: 196-201.

9. Warkentin T.E., Levine M.N., Hirsh J., et al. Heparin-induced thrombocytopenia in patients treated with low-molecular-weight beparin or unfractionadet heparin. N Engl J Med 1995; 332 (20): 1330-5.

10.Wunderle V.H., Kainer K., Salzmann G., et al. Heparin-induced thrombosis despite normal platelet counts in vascular surgery.

Am J Surg 1997; 173: 117-9.

11. Chong B.H., Eisbacher M. Pathophysiology and laboratory testing of heparin-induced thrombocytopenia. Semin Hematol 1998; 35 (4 suppl 5): 3-8.
12. Greinacher A., Janssens U., Berg G., et al. Lepirudin (recombinant birudin) for parenteral anticoagulation in patients with beparin-induced thrombocytopenia. Circulation 1999; 100: 587-93.

13. Greinacher A., Völpel H., Janssens U., et al. Recombinant hirudin (lepirudin) provides safe and effective anticoagulation in patients with heparin-induced thrombocytopenia. A prospective study. Circulation 1999; 99: 7380.

14. Warkentin T.E. Limitations of conventional treatment options for heparin-induced thrombocytopenia. Semin Hematol 1998; 35 (4 suppl 5): 17-25.

15. Ortel T.L., Chong B.H. New treatment options for heparin-induced thrombocytopenia. Semin Hematol 1998; 35 (4 suppl 5): 2634.

16. Warkentin T.E., Kelton J.G. Thrombocytopenia due to platelet destruction and hypersplenism. In: Hoffman R, Benz Jr EJ, Shattil SJ, et al. Hematology basic principles and practice. 3th ed. Philadelphia: Churchill Livingstone, 2000. p. 2138-54.

17. Furie. Oral anticoagulant therapy. In: Hoffman R, Benz Jr EJ, Shattil SJ, et al. Hematology basic principles and practice. 3th ed. Philadelphia: Churchill Livingstone, 2000. p. 2040-6.

Recebido: 11/01/01

Aceito: 30/05/01 